\title{
Structures in the energy distribution of the scission neutrons: finite neutron-number effect
}

\author{
N. $\operatorname{Carjan}^{1,2,3 *}$ and M. Rizea ${ }^{1}$ \\ 1 "Horia Hulubei" National Institute of Physics and Nuclear Engineering, Bucharest-Magurele, RO-07r125, Romania \\ ${ }^{2}$ Joint Institute for Nuclear Research, FLNR, 141980 Dubna, Moscow Region, Russia \\ 3 CENBG, University of Bordeaux, 33175 Gradignan, France
}

(Dated: November 15, 2018)

\begin{abstract}
The scission neutron kinetic energy spectrum is calculated for ${ }^{236} U$ in the frame of the dynamical scission model. The bi-dimensional time dependent Schrödinger equation with time dependent potential is used to propagate each neutron wave function during the scission process which is supposed to last $1 \times 10^{-22}$ sec. At the end, we separate the unbound parts and continue to propagate them as long as possible (in this case $50 \times 10^{-22} \mathrm{sec}$ ) in the frozen fragments approximation. At several time intervals, the Fourier transforms of these wave packets are calculated in order to obtain the corresponding momentum distributions which lead to the kinetic energy distributions. The evolution of these distributions in time provides an interesting insight into the separation of each neutron from the fissioning system and asymptotically gives the kinetic energy spectrum of that particular neutron. We group the results in substates with given projection $\Omega$ of the angular momentum on the fission axis to study its influence on the spectrum. Finally, the sum over all $\Omega$ values is compared with a typical evaporation spectrum as well as with recent precise measurements in the reaction ${ }^{235} U\left(n_{t h}, f\right)$. Structures are present both in the scission-neutron spectrum and in the data.

Keywords: scission neutron, dynamical model, Fourier transform in cylindrical coordinates, kinetic energy spectrum
\end{abstract}

\section{INTRODUCTION}

It is now generally accepted that prompt fission neutrons (PFN) have two components with unknown relative intensities. In chronological order, these components are: neutrons dynamically released at scission (SN) and neutrons evaporated from fully accelerated fragments $(\mathrm{EVN})$. There is no indication which of these two components is the dominant one since the gross features of PFN can be reproduced by both models [1 10]. To determine the relative percentage of $\mathrm{SN}$ and $\mathrm{EVN}$, instead of looking at averaged properties, one has to analyze PFN observables correlated with fragment properties in order to remove the above mentioned ambiguity.

It is also important to find differences, even small, between the predicted properties of the scission and evaporated neutrons that may be investigated experimentally, thus making the separation of the two components possible. It has been already pointed out [4] that, for a fixed fragment-mass division, the angular distributions with respect to the fission axis of EVN and of SN are different: the first is smooth while the second presents oscillations due to the proximity of the fragments at the moment of emission.

This time we concentrate on the kinetic energy spectrum of the scission neutrons, again for a given fragmentmass ratio. We calculate it for neutrons with quantum numbers $\Omega=1 / 2,3 / 2,5 / 2,7 / 2$ and $9 / 2 . \Omega$ is the projection of the angular momentum on the fission axis. They

\footnotetext{
* carjan@theory.nipne.ro
}

account for $99 \%$ of the total multiplicity. The result is compared with a typical evaporation spectrum to reveal differences.

Sec 2 contains the description of the model used. The corresponding equations are given in Sec 3. Numerical results for individual neutron states in ${ }^{236} U$ are presented in Sec 4. In Sec 5 the total energy spectrum is calculated and compared with recent measurements. The summary is in Sec 6.

\section{FROM BOUND TO FREE NEUTRONS}

In order to calculate the kinetic energy spectrum of the scission neutrons we need to identify the part of each neutron wave packet which left the fissioning system and therefore represents a free neutron.

We do this in the frame of the dynamical scission model [11] in which the fissioning system undergoes a diabatic transition during the neck rupture. Due to the coupling with the rapidly changing potential, each initially bound neutron state becomes a wave packet with few components in the continuum. This process is simulated introducing a time-dependent potential (TDP) in the two-dimensional time-dependent Schrödinger equation (TDSE2D). The model is best suited to low energy fission: spontaneous or sub-barrier. An amount of excitation energy at the last saddle point could lead to a neutron evaporation before scission which is not included in the present calculations.

There are three parameters in the dynamical scission model: the nuclear shapes just before $\left(\alpha_{i}\right)$ and immediatelly after scission $\left(\alpha_{f}\right)$ and the duration $\Delta T$ of the tran- 
sition between these two shapes. These quantities are not really known; one can only make educated guesses about them. The lower limit of $\Delta T$ should be about $5 \times 10^{-23}$ sec i.e., the time required for a Fermi level nucleon to cross a $4 \mathrm{fm}$ thick neck. A value of $\Delta T$ between 1 and $2 \times 10^{-22}$ sec can therefore be considered realistic. The minimum neck radius $r_{\text {min }}$ in the initial configuration predicted by the optimal scission shapes [12] is $\approx 2 \mathrm{fm}$. It is a generally accepted value since it can be deduced also from general considerations like the size of the alpha particle. We take a slightly lower value $(1.6 \mathrm{fm})$. There is no indication about the minimum distance between the surfaces of the two fragments $d_{\min }$ in the final configuration. We take $0.6 \mathrm{fm}$. These $r_{\min }$ and $d_{\min }$ values were already used in our first publication [13] and have never been changed. They lead to an average scission neutron multiplicity of 0.6 neutrons per fission event, i.e., to only $25 \%$ of the total prompt fission neutron multiplicity. Although we know [1] that both $\left\langle\nu_{s c}\right\rangle$ and the average kinetic energy $\left\langle E_{k i n}>\right.$ are sensitive to the the parameters of the model, we do not think that it makes sense to adjust them to the existing experimental values for all prompt fission neutrons (i.e., to 2.41 and $1.99 \mathrm{MeV}$ respectively, obtained in the reaction $\left.{ }^{235} U\left(n_{t h}, f\right)\right)$. When more reliable values for these quantities are available we will use them and find out how significant is the percentage of neutrons released at scission and emitted during the acceleration of the fragments. In fact, self-consistent microscopic models, such as the density functional theory extended to superfluid systems and real-time dynamics [14], could provide estimates for the three parameters of our model.

The unbound components of the neutron wave packet will start leaving the nascent fragments immediately after scission but this separation takes time. Hence they leave during the acceleration phase: up to approximately $6 \times 10^{-21} \mathrm{sec}$ for most of them. This is a rough estimation based on the half-live of neutron emission at scission which is about $2 \times 10^{-21} \sec$ [11] if $\Omega=1 / 2$. Large times require large spatial grids. Although we implemented transparent boundary conditions [15], the reflexions on the boundaries of the numerical grid are not completely reduced and we need to push our computational resources to their limit.

At the beginning, i.e., immediately after scission, the unbound neutrons are mainly localized inside the nucleus and therefore possess very high kinetic energies (of the order of the depth of the potential). To obtain the measured spectrum, one has to wait until these neutrons are outside the fissioning system. This detachment is simu- lated with TDSE2D, using a constant potential this time. We stop at $T_{\max }=5 \times 10^{-21} \mathrm{sec}$ when the percentage of unbound neutrons that are still inside the nucleus attains a minimum (about 10\%).

Since the neutron motion is much faster than the separation of the nascent fragments, the freeze of the fissioning nucleus at its configuration immediately after scission is justified and it simplifies our numerical task. Even when the neutrons are outside the fragments, their kinetic energy is at least $1.5 \mathrm{MeV}$ (see Figs. 1 to 8 ). The total kinetic energy of the fully accelerated fragments is $0.75 \mathrm{MeV} /$ nucleon on the average. Therefore, at the begining of the acceleration phase when the scission neutrons are emitted, the velocity of the fragments is negligible as compared with the velocity of the neutrons.

The Fourier transforms of the unbound-neutron wave packets give, at each time, the momentum distributions and therefore also the kinetic energy distributions. Asymptotically, the sum over all neutrons, weighted with their occupation probabilities, leads to the scission neutron spectrum.

Let us now put the description from above into equations.

\section{FORMALISM}

The scission consists in the neck rupture and the absorption of the neck stubs by the nascent fragments. We consider axially symmetric fissioning nuclei and use cylindrical coordinates. Let $\left|\Psi^{i}(\rho, z)\right\rangle$ be the eigenfunctions of the Hamiltonian of independent neutrons in the justbefore-scission configuration. During the scission process these functions evolve in a time-dependent potential according to TDSE2D:

$$
i \hbar \frac{\partial \Psi^{i}(\rho, z, t)}{\partial t}=\mathcal{H}(\rho, z, t) \Psi^{i}(\rho, z, t) .
$$

The solution is obtained using a numerical scheme of Crank Nicolson type [16, 17]. The infinite physical domain is replaced by a finite grid: $\left[0, \rho_{\max }\right] \times$ $\left[-z_{\operatorname{maz}}, z_{\max }\right]=[0,84 \mathrm{fm}] \times[-128 \mathrm{fm}, 128 \mathrm{fm}]$ with $\Delta \rho=$ $\Delta z=1 / 8 \mathrm{fm}$. For the time evolution we use a step $\Delta t=1 / 128 \times 10^{-22}$ sec. Special conditions on the boundaries of the grid are imposed to reduce reflexions [15].

In the non-adiabatic regime, the propagated wave functions $\left|\Psi^{i}(\rho, z, t)\right\rangle$ are wave packets which have also positive-energy components.

The probability amplitude that a neutron occupying the state $\left|\Psi^{i}\right\rangle$ before scission populates an eigenstate $\left|\Psi^{f}\right\rangle$ immediately-after scission is

$$
a_{i f}=\left\langle\Psi^{i}(\Delta T) \mid \Psi^{f}\right\rangle=2 \pi \iint\left(f_{1}^{i}(\Delta T) f_{1}^{f}+f_{2}^{i}(\Delta T) f_{2}^{f}\right) \rho d \rho d z
$$

$a_{i f}$ is $\neq 0$ only if $\left|\Psi^{i}\right\rangle$ and $\left|\Psi^{f}\right\rangle$ have the same projection
$\Omega$ of the total angular momentum. $\Delta T$ is the duration 
of the scission process assumed here to be $10^{-22}$ sec i.e., relatively short. $f_{1}$ and $f_{2}$ are the two components of the wave function corresponding to spin up and spin down respectively.

The probability that this neutron is unbound at the end of the scission process is given by:

$$
P_{e m}^{i}=v_{i}^{2}\left(\sum_{\text {unbound }}\left|a_{i f}\right|^{2}\right)=v_{i}^{2}\left(1-\sum_{\text {bound }}\left|a_{i f}\right|^{2}\right)
$$

where $v_{i}^{2}$ is its initial occupation probability.

The part of the wave packet which is in the continuum at $\Delta T$ :

$$
\left|\Psi_{e m}^{i}\right\rangle=\left|\Psi^{i}(\Delta T)\right\rangle-\sum_{\text {bound }} a_{i f}\left|\Psi^{f}\right\rangle .
$$

will leave the fissioning nucleus and asymptotically will describe the emitted scission neutron.

To calculate the scission neutron spectrum we have therefore to propagate $\left|\Psi_{e m}^{i}\right\rangle$ for as long as possible, let's say until $\Delta T+T_{\max }$ with $T_{\max }=50 \times 10^{-22} \mathrm{sec}$. Since the separation of the fragments is slower than the neutron emission, for the sake of simplicity, we keep the fragments in their configuration at $\Delta T$.

In order to vizualize the detachment of the unbound fractions of the neutron wave packets from the fissioning system, we extract at several times $\Delta T+T$ these fractions and calculate their Fourier transform [18, 19]:

$$
F^{i}\left(k_{\rho}, k_{z}, T\right)=2 \pi \int_{-\infty}^{\infty}\left[\int_{0}^{\infty} \Psi_{e m}^{i}(\rho, z, T) J_{0}\left(2 \pi \rho k_{\rho}\right) \rho \mathrm{d} \rho\right] \mathrm{e}^{-2 \pi \mathrm{i} z k_{z}} \mathrm{~d} z
$$

In this way we can study the probabilities both in coordinate and in momentum space as a function of time. $J_{0}$ is the zero-order Bessel function of the first kind. The transform with respect to the variables $\rho, k_{\rho}$ is called the zero-order Hankel transform. Thus, the Fourier transform in cylindrical coordinates implies a combination of Hankel and one-dimensional Fourier transforms. The present study represents the first application in nuclear physics of such transforms.

\section{POST-SCISSION EVOLUTION OF THE UNBOUND NEUTRONS AND OF THEIR KINETIC ENERGIES}

Calculations are performed for the fission of ${ }^{236} U$ having in mind the reaction ${ }^{235} U\left(n_{t h}, f\right)$ which has been remeasured recently with better statistics and improved resolutions in mass, angle and energy [20, 21]. The preand post-scission nuclear shapes are described by Cassini ovals 22] with only two parameters corresponding to the overall elongation and the mass asymmetry [23]. Numerical results for the most probable mass division (light fragment mass $A_{L}=96$ ) are presented.

We have calculated the Fourier transform using Eq. (5) for wavefunctions corresponding to $\Omega=1 / 2,3 / 2,5 / 2$, $7 / 2$ and $9 / 2$. Each point in the $\left(k_{\rho}, k_{z}\right)$ plane corresponds to an absolute value $K=\sqrt{k_{\rho}^{2}+k_{z}^{2}}$ and a probability $P=\left|F\left(k_{\rho}, k_{z}\right)\right|^{2} k_{\rho} \Delta k_{\rho} \Delta k_{z}$ that a scission neutron has its momentum $\vec{K}$ in the volume element $\mathrm{d}^{3} \vec{K}$. The points of constant K-value lie on a circle. Since the Fourier transform is given only on the grid points we can represent the $K$-distribution only as a histogram. For this we divide the domain of $K$-values in equal intervals and group the grid points according to the interval to which they belong.
Summing up the probabilities of the points in each group one obtains the probability $P_{i}(K)$ that a given neutron $i$ has its $K$-value in the respective interval. From the momentum distribution one can deduce the kinetic energy distribution, $P_{i}\left(E_{k i n}\right)$, using the relation $E=\frac{\hbar^{2}}{2 \mu} K^{2}$ and multiplying with the Jacobian $d E / d K \sim E^{1 / 2}$ in order to accommodate for this change of variable.

In the figures 1 to 3 are shown unbound wave packets for $\Omega=1 / 2$ and indices $i=22,26$ and 28 (as sum of square moduli of the two components $f_{1}$ and $f_{2}$ ) juxtaposed with kinetic-energy histograms at different times $T$ after scission.

The initial wave packets are given by Eq.(4). At $T=0$, i.e. immediately after scission, the released neutron populates bound states in the continuum and it is mainly localized in the neck region since it is there that the potential changes mostly. The kinetic energy of the unbound neutron can reach values as high as the potential depth $V_{0}$ which is $40.2 \mathrm{MeV}$ in our case. The average value is however lower (around $30 \mathrm{MeV}$ ) due a large diffuse surface and tails of the wave functions that penetrate into the potential wall. One notices that with increasing time ( $T=20$ and $50 \times 10^{-22} \mathrm{sec}$ ) the amplitude of the wavefunctions diminishes, showing that the neutron is leaving the nucleus. At the same time, the $E_{k i n}$-distribution is shifted to lower values, reflecting the fact that the neutrons are less and less present inside the potential well.

At very large times the neutron should be completely emitted. One sees that, due to numerical limitations, we cannot reach this situation: even at $T_{\max }$ the neutron has still $10 \%$ probability to be inside the fragments. If we calculate longer, the part of the wave packet that is reflected on the boundary of the spatial grid returns inside the nucleus affecting the energy spectrum. $T_{\max }$ is therefore related to the size of the $(\rho, z)$ grid used. 

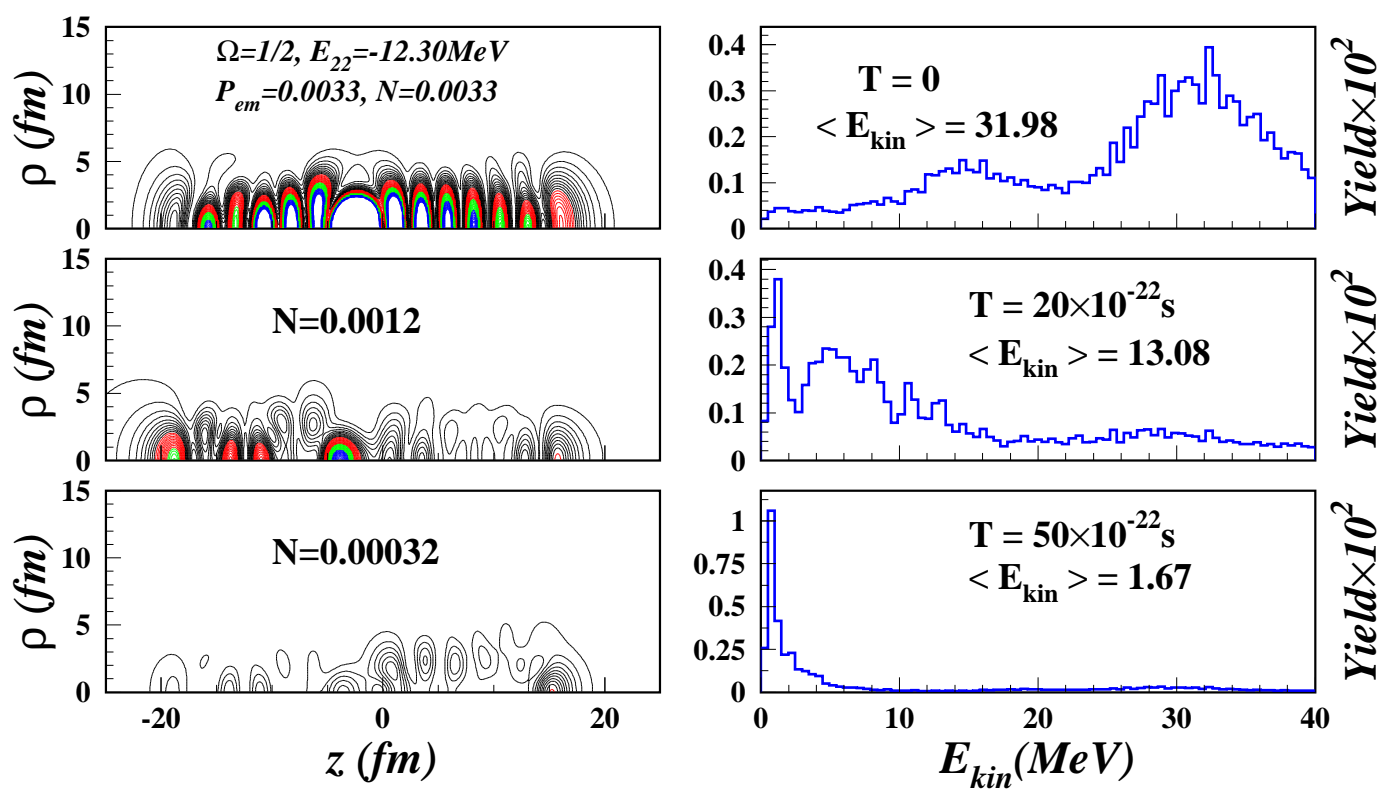

FIG. 1. Square modulus of the unbound $\mathrm{WF}_{22}$ (left column) and energy distribution (right column) at different times T. The wavefunctions at $T=0$ and $50 \times 10^{-22} \mathrm{sec}$ are represented relative to that at $T=20 \times 10^{-22}$ sec. The values on the ordinates of the histograms are $P_{22}\left(E_{k i n}\right)$ probabilities multiplied by $10^{2} . E_{k i n}^{\text {mean }}=\frac{\sum_{m, n} E_{k i n} P E_{k i n}^{1 / 2}}{\sum_{m, n} P E_{k i n}^{1 / 2}}$ where $P=k_{\rho}|F|^{2} d k_{\rho} d k_{z}$.

$\mathrm{N}$ is the probability that the wave function is inside the nucleus at a given time $\mathrm{T}$.
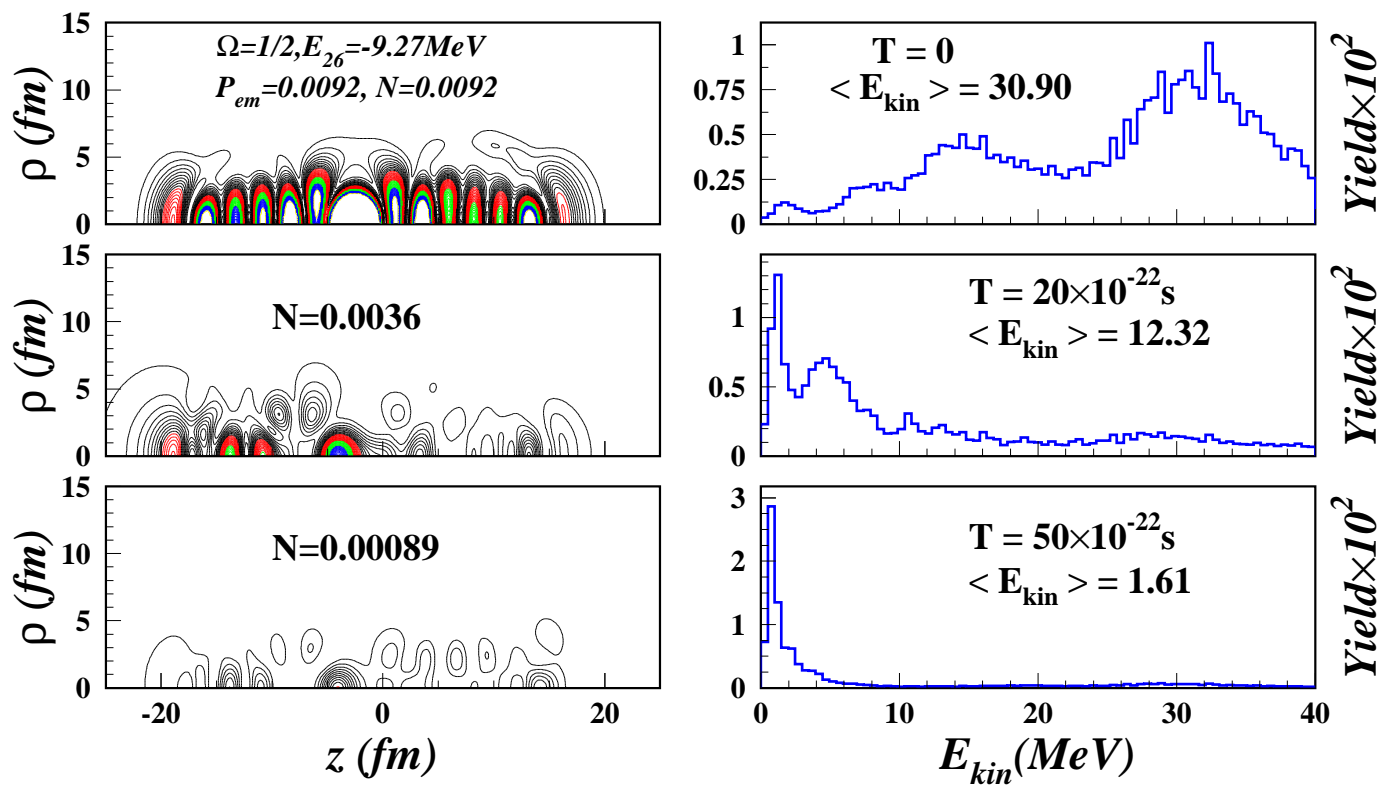

FIG. 2. The same as in Fig.1 but for the unbound $\mathrm{WF}_{26}$.

Since the above mentioned probability is small, one can consider that at $T=50 \times 10^{-22} \mathrm{sec}$ the calculated $E_{k i n^{-}}$ distribution represents well the emitted neutron. These single spectra are characterized by a peak at low energies (below $2 \mathrm{MeV}$ ) plus a short tail towards higher energies.

So far we have analyzed energy distributions for wave functions with $\Omega=1 / 2$ which correspond to orbital angular momentum projections $\Lambda=0$ or 1 . In most cases 

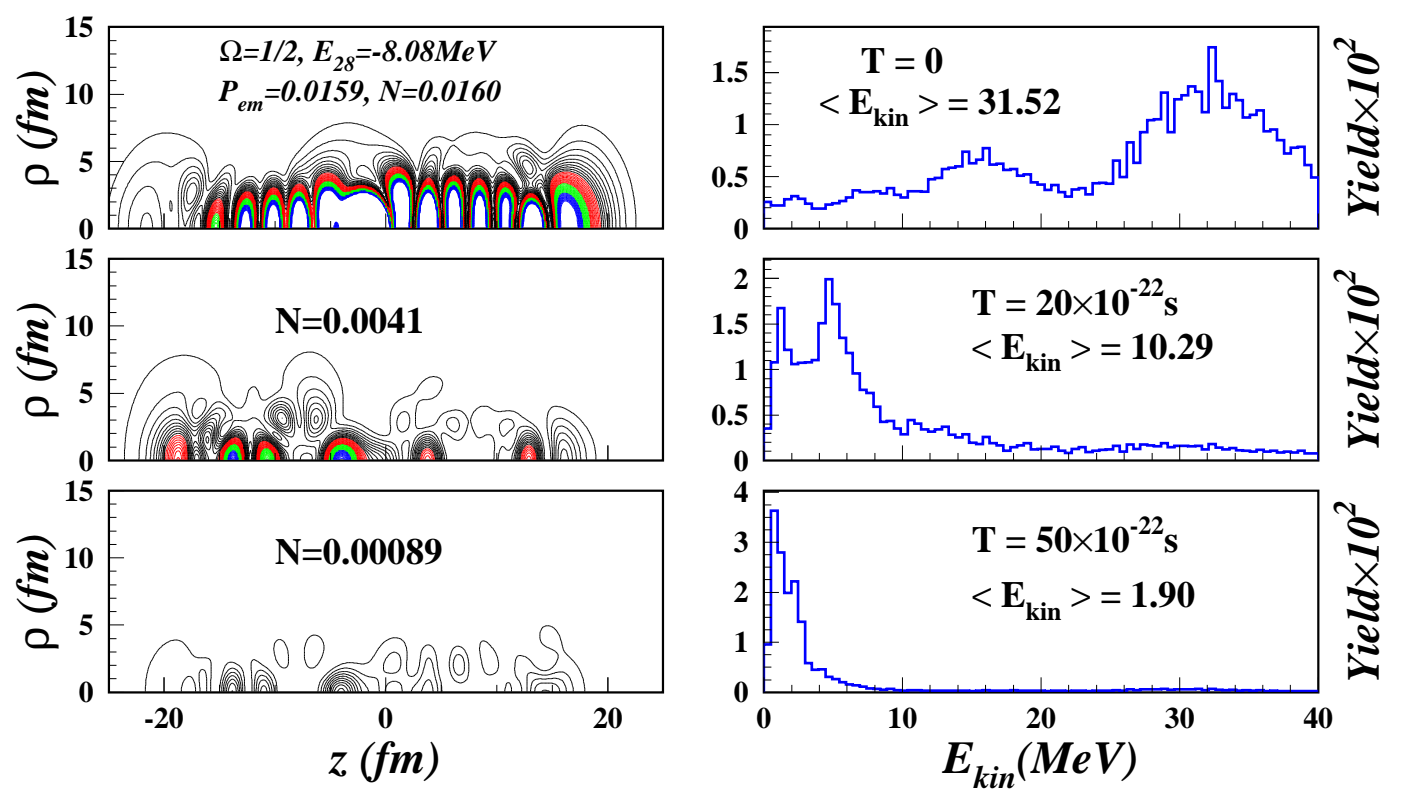

FIG. 3. The same as in Fig.1 but for the unbound $\mathrm{WF}_{28}$.
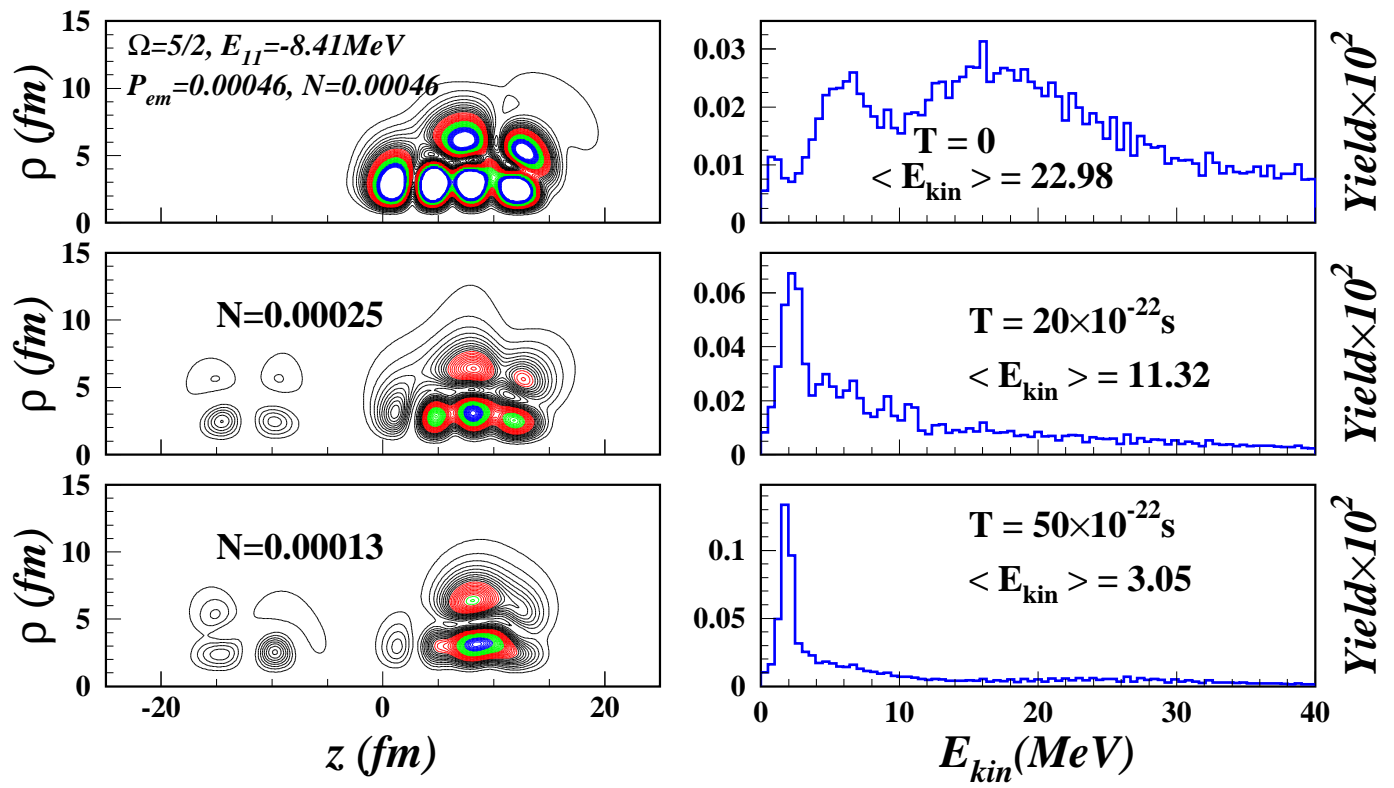

FIG. 4. Square modulus of the unbound $\mathrm{WF}_{11}^{5 / 2}$ (left column) and energy distribution (right column) at different times T. The projection of the angular momentum on the fission axis of this wave function is $\Omega=5 / 2$. The wavefunctions at $T=0$ and $50 \times 10^{-22}$ sec are represented relative to that at $T=20 \times 10^{-22} \mathrm{sec}$. The values on the ordinates of the histograms are $P_{11}\left(E_{k i n}\right)$ probabilities multiplied by $10^{2} . E_{k i n}^{m e a n}=\frac{\sum_{m, n} E_{k i n} P E_{k i n}^{1 / 2}}{\sum_{m, n} P E_{k i n}^{1 / 2}}$ where $P=k_{\rho}|F|^{2} d k_{\rho} d k_{z}$.

$\mathrm{N}$ is the probability that the wave function is inside the nucleus at a given time $\mathrm{T}$.

$\left|f_{1}\right|^{2}>>\left|f_{2}\right|^{2}$, see Eq.(2), so there is practically no centrifugal barrier.

However, for larger $\Omega$ values, the centrifugal potential,
$\Lambda_{1,2}^{2} / \rho^{2}$, is expected to play a role. In the figures 4 to 6 are shown emitted wave function with $\Omega=5 / 2(\Lambda=2$ or 3$)$ and indices 11, 12 and 13 and the corresponding kinetic 

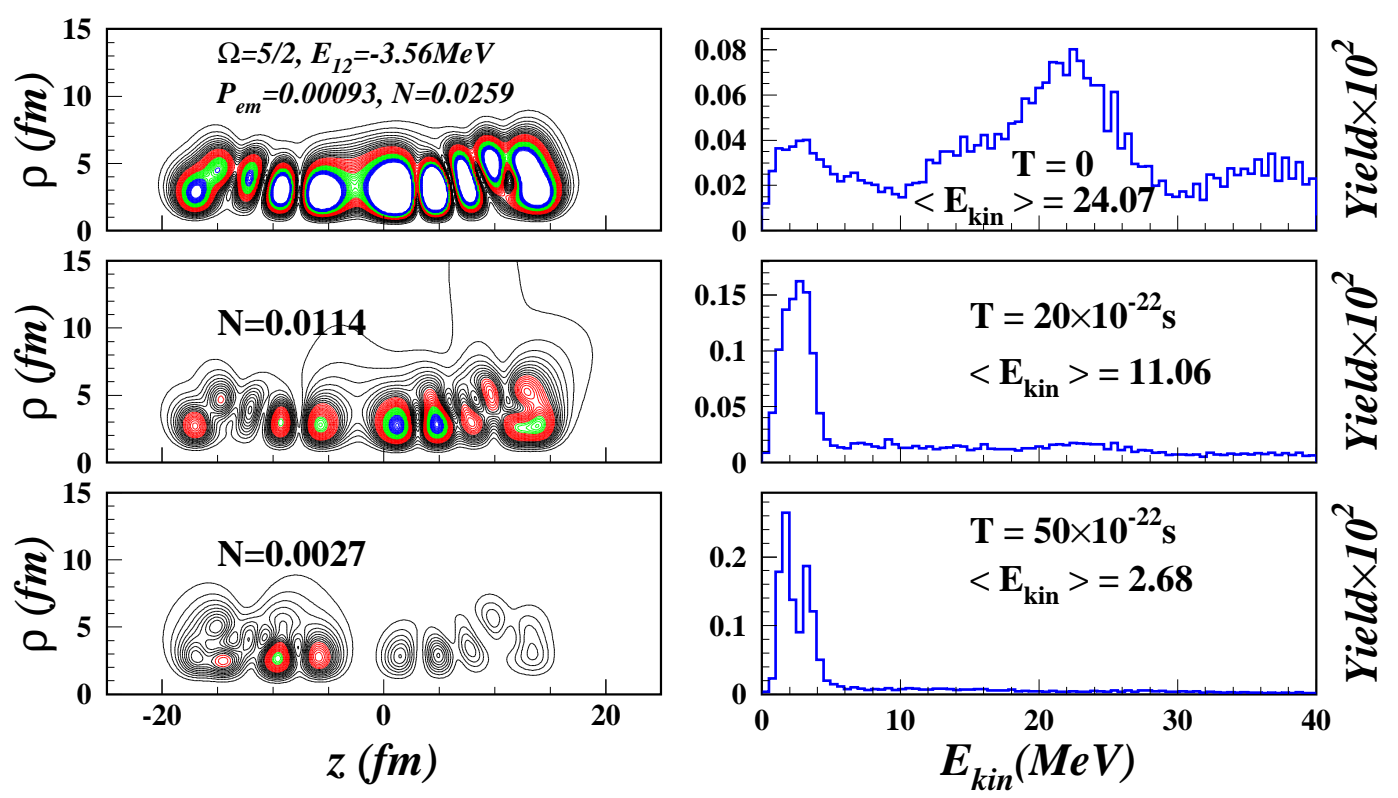

FIG. 5. The same as in Fig.4 but for the unbound $\mathrm{WF}_{12}^{5 / 2}$.
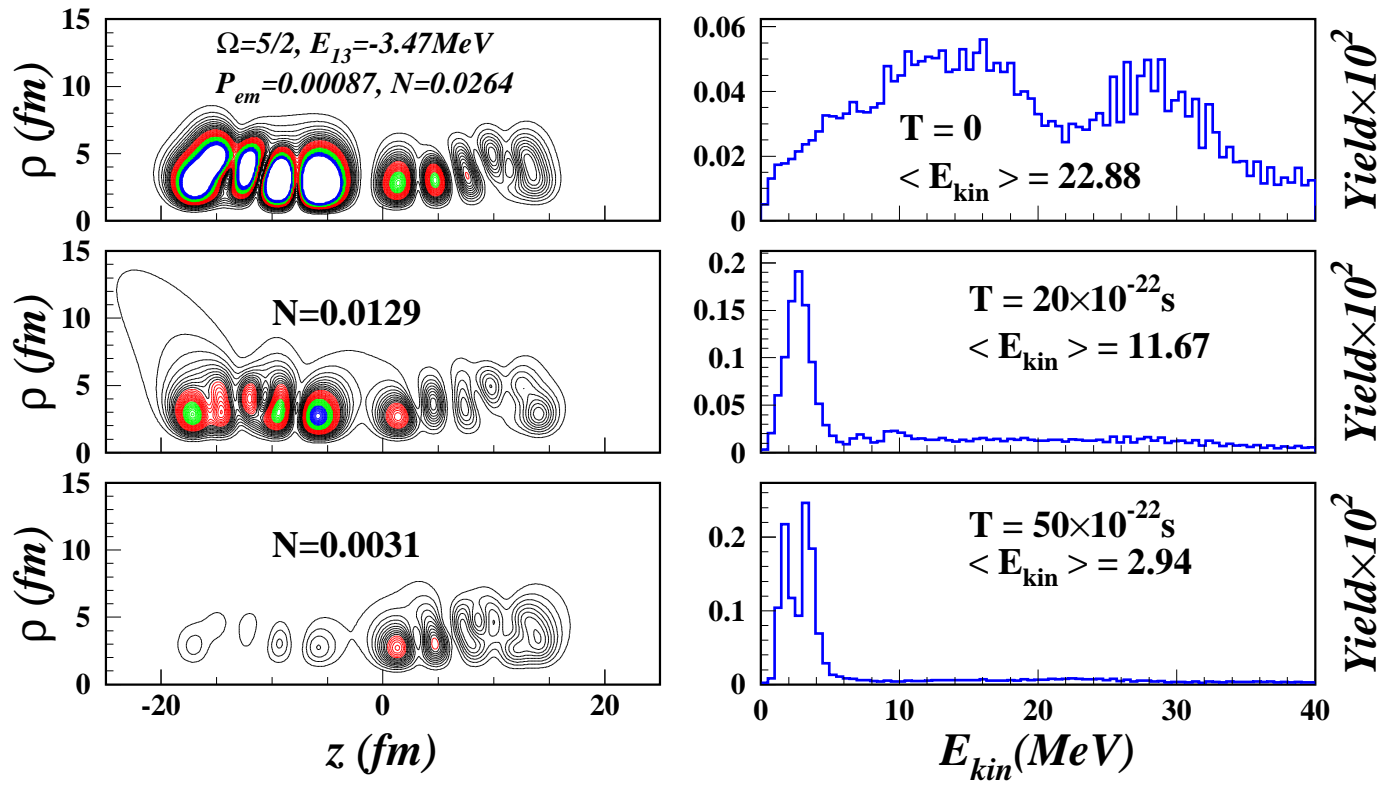

FIG. 6. The same as in Fig.4 but for the unbound $\mathrm{WF}_{13}^{5 / 2}$.

energy histograms. As compared with the previous case:

a) At $T=0$ the square moduli of the unbound wave functions, are displaced from the $\mathrm{z}$ axis where the centrifugal potential has a maximum. Of course this comes from the same feature of the total wave functions. For this reason they are less present in the neck region and contribute less to the scission neutron multiplicity.

b) At $T=0$ the spectrum is shifted towards lower values since the kinetic energy is reduced by the centrifugal potential. As a result the average kinetic energy is smaller $(\approx 23 \mathrm{MeV})$. At $T=50 \times 10^{-22} \mathrm{sec}$ the average kinetic energy is larger $(\approx 3 \mathrm{MeV})$ since the centrifugal potential is now transformed into kinetic energy.

Figs. 7 and 8 show the time evolution of the wave packets and of the kinetic energy histograms for states (indices 4 and 6) corresponding to an even higher $\Omega$ value 

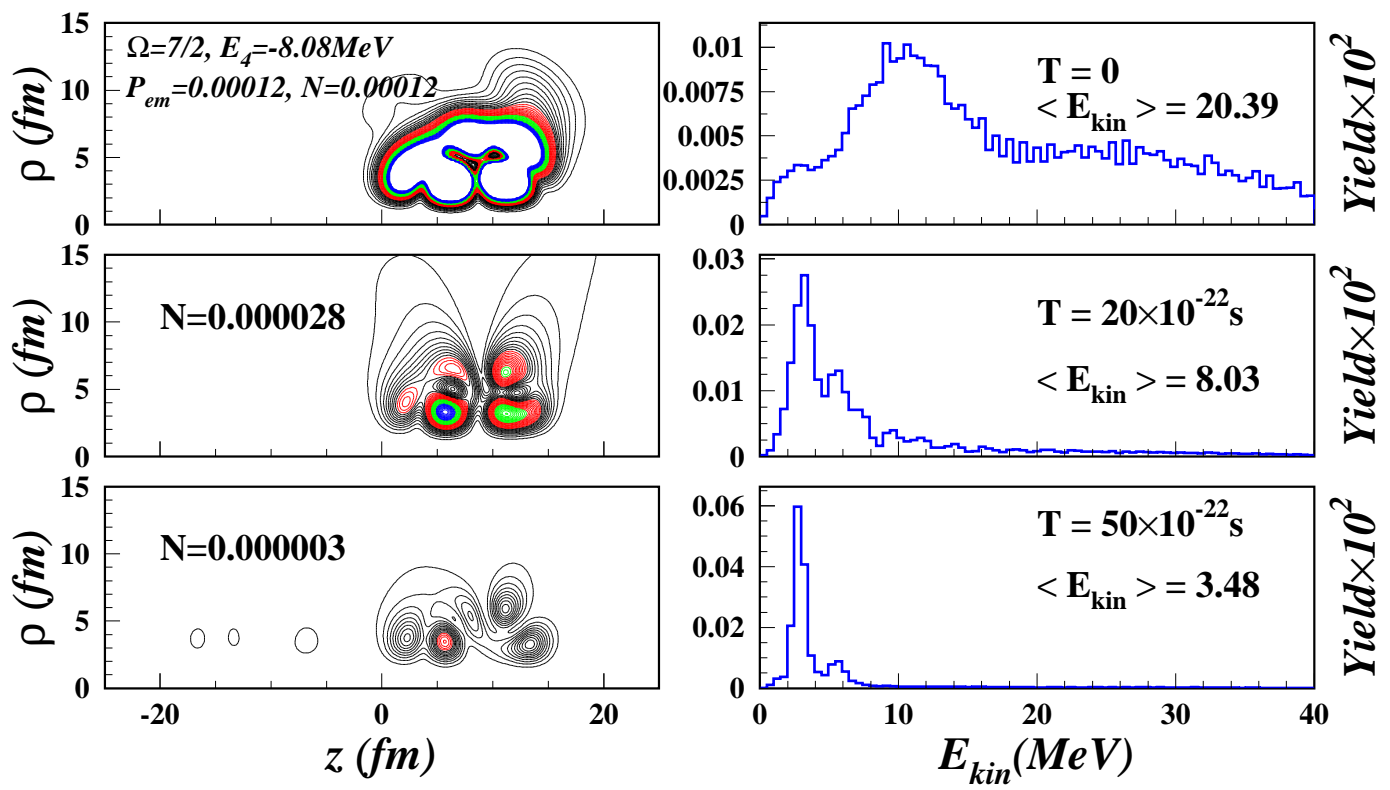

FIG. 7. Square modulus of the unbound $\mathrm{WF}_{04}^{7 / 2}$ (left column) and energy distribution (right column) at different times T. The projection of the angular momentum on the fission axis of this wave function is $\Omega=7 / 2$. The wavefunctions at $T=0$ and $50 \times 10^{-22} \mathrm{sec}$ are represented relative to that at $T=20 \times 10^{-22} \mathrm{sec}$. The values on the ordinates of the histograms are $P_{04}\left(E_{k i n}\right)$ probabilities multiplied by $10^{2} . E_{k i n}^{m e a n}=\frac{\sum_{m, n} E_{k i n} P E_{k i n}^{1 / 2}}{\sum_{m, n} P E_{k i n}^{1 / 2}}$ where $P=k_{\rho}|F|^{2} d k_{\rho} d k_{z}$.

$\mathrm{N}$ is the probability that the wave function is inside the nucleus at a given time $\mathrm{T}$.
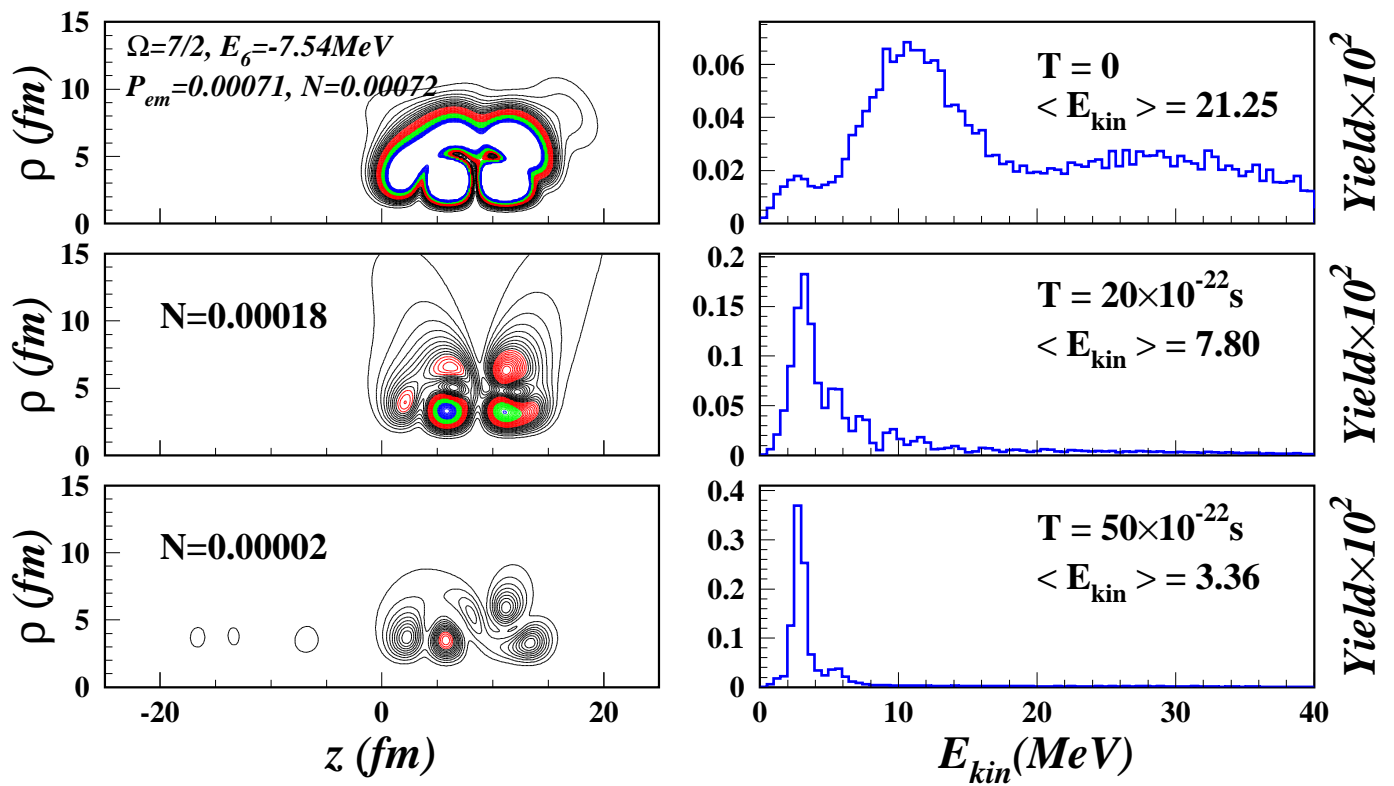

FIG. 8. The same as in Fig.7 but for the unbound $\mathrm{WF}_{6}^{7 / 2}$.

$(7 / 2)$. As expected, $<E_{k i n}>$ becomes even lower $(\approx 21$ $\mathrm{MeV})$ at $T=0$ and even larger $(\approx 3.5 \mathrm{MeV})$ at $T=50 \times$ $10^{-22} \mathrm{sec}$. An interesting feature of the individual spectra at high $\Omega$ values is the existence of a peak at low energies in the initial spectrum, the intensity of which increases with time. It reflects the fact that the wave functions, 
being located at the nuclear surface, spread outside the fragments even at $T=0$.

\section{SCISSION NEUTRON SPECTRUM}

To obtain the whole kinetic energy spectrum for a fixed mass asymmetry, one has to sum the single spectra over all occupied states and all $\Omega$ value.

The upper five frames of Fig. 9 show kinetic energy spectra for $\Omega=1 / 2, \Omega=3 / 2, \Omega=5 / 2, \Omega=7 / 2$ and $\Omega=9 / 2$ respectively. The kinetic energy increases with increasing $\Omega$ due to the the centrifugal term. Note however that $\Omega=1 / 2$ gives the dominant contribution (65\%).

In the 6 th frame, the total spectrum (summed over the five $\Omega$ values) is shown. It presents a maximum around $0.7 \mathrm{MeV}$ and an exponentially decreasing tail till $8 \mathrm{MeV}$ in qualitative agreement with the measured spectrum [24] of all prompt fission neutrons (PFN). For comparison, we added recent data [20] obtained for the same constraint on mass asymmetry $\left(A_{L}=96\right)$. One notices that both the data and the calculation are not smooth. The oscillations in the data are statistically significant. The calculated distribution is not smooth since it consists of a finite weighted sum of individual contributions with different mean values and widths. The number of non-negligible terms is only 35, distributed among the $\Omega$ values as following: 21 for $1 / 2,8$ for $3 / 2,4$ for $5 / 2$ and 2 for $7 / 2$. Hence less than half of the total number of the neutrons in ${ }^{236} U$ contribute significantly to the scission neutron spectrum.

However the data do not oscillate as much as the calculations. One reason is that the data are affected by a finite energy resolution. If we convolute the theoretical spectrum with a Gaussian resolution function, the amplitude of its oscillations will decrease. The other reason is that our model (as any model) contains approximations and numerical limitations.

Two typical evaporation spectra [25], $E \exp (-E /$ Temp $)$, for nuclear temperatures Temp $=1.0$ and $0.9 \mathrm{MeV}$ are also ploted. We stress that, in this case $\left(A_{L}=96\right)$, each fragment evaporates about one neutron on the average and the Weisskopf formula should work. These evaporation spectra follow quite well the general trend of the recent data except at very low and very high energies. Temp $=0.9 \mathrm{MeV}$ reproduces better the drop at low energies while $T e m p=1.0 \mathrm{MeV}$ the tail at high energies. Evidently, none of them exhibit oscillations.

In the lowest frame of Fig. 9 the same comparison is shown in lin-log scale to unveil hidden differences at $E_{k i n}>5 \mathrm{MeV}$. One can see that, in contrast to the EVN, the SN can reproduce the high energy tail of the PFN spectrum. This inability of the evaporation hypothesis to account for high energy PFN has been already discussed in Ref. [26].

\section{SUMMARY}

The dynamical scission model [11] is used to calculate SN kinetic energy spectra, at different intervals of time after scission, for the fission of ${ }^{236} U$ into the most probable mass division $\left(A_{L}=96\right)$. The evolution of the wave packets $\left|\Psi_{\text {em }}^{i}\right|^{2}$ (representing the neutrons released during scission) and of their kinetic energy, $E_{k i n}$, distributions reflects the process of separation of the scission neutrons from the nascent fission fragments.

The spectrum at the largest time we were able to attain numerically (i.e., $T_{\max }=5 \times 10^{-21} \mathrm{sec}$ ) is compared with recent measurements obtained with high statistics and resolution [20] in the reaction ${ }^{235} U\left(n_{t h}, f\right)$ for the same mass division. As in the case of the PFN angular distribution [1, 4], both hypotheses (evaporation from fully accelerated fragments and dynamical emission at scission) explain satisfactorily the general features of the measured spectrum. This difficulty to distinguish experimentally between two completely oposite theoretical assumptions is puzzling.

There is however a detail that makes the results of the two hypotheses slightly different: the evaporation spectrum is smooth while the SN spectrum presents structures due to the finite number of neutrons that contribute.

In spite of computational limitations (not large enough $(\rho, z)$ grid and not long enough time evolution), a more quantitative agreement could be forseen by including the simultaneous separation of the fragments after scission and by taking into account the re-absorption of the unbound neutrons by the imaginary potential of the nascent fragments. Such calculations are in progress.

\section{ACKNOWLEDGEMENTS}

This work was done in the frame of the projects: PNIII-P4-ID-PCE-2016-0649 (contract nr. 194/2017), PCE2016-0014 (contract nr.7/2017) and EC EURATOM FP7 project CHANDA.

\section{Appendix: Total neutron wave packets and their emitted parts at $\alpha_{f}$}

As stated in Sec 2, after a diabatic transition at scission, all neutrons are represented by expansions in the set of eigenstates of the nuclear configuration $\alpha_{f}$. At the higher end, these wave packets are built on states in the continuum which can therefore leave the nucleus. In the dynamical scission model these small parts, defined by Eq. (4), are the scission neutrons. From pedagogical point of view it is useful to vizualize and understand the differences between the total wave packet and its tiny unbound tail.

In the figures 10-12 are shown three wave packets corresponding to $\Omega=1 / 2$. For states with low energies 


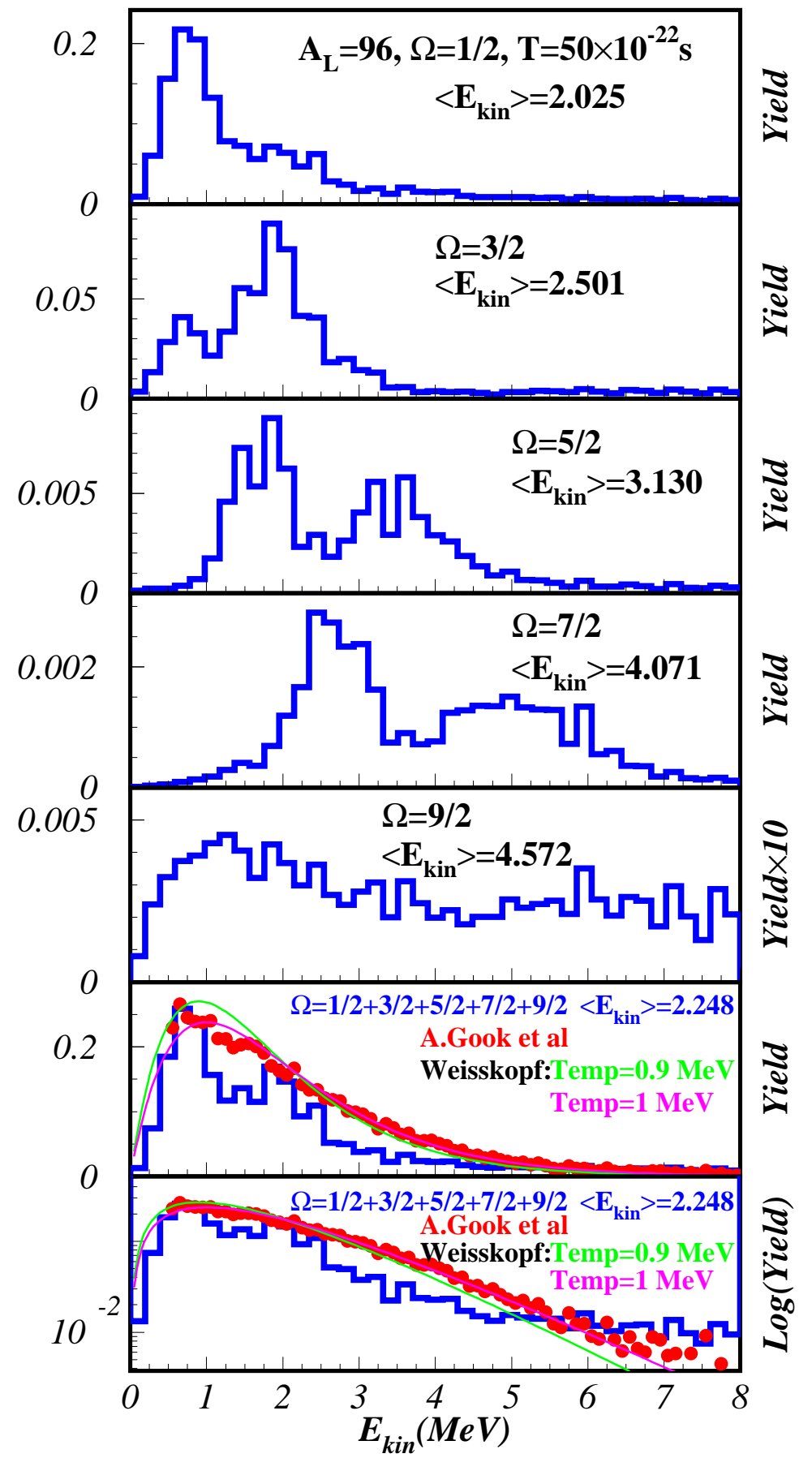

FIG. 9. Kinetic energy distributions at $T=50 \times 10^{-22}$ sec for sub-states defined by the quantum number $\Omega$. In the two lowest frames the distribution calculated with all neutron states is presented together with recent experimental results 20] from the reaction ${ }^{235} U\left(n_{t h}, f\right)$. Two typical evaporation spectra [25] characterized by nuclear temperatures Temp = 1.0 and $0.9 \mathrm{MeV}$ are also plotted for comparison. The evaporation spectra were normalized to the data but there is an arbitrary normalization between the data and the calculation. $\left(\right.$ Yield $\left.=\sum P_{i}\left(E_{k i n}\right) \times v_{i}^{2}\right)$. 
the total wave functions $\left(\mathrm{WF}_{13}\right.$ and $\left.\mathrm{WF}_{14}\right)$ are confined in one of the fragments (light or heavy). The emitted wave functions are concentrated in the neck region where the coupling to the changing potential is the strongest. For states with high energies, the total wave functions are localized in both fragments (like $\mathrm{WF}_{28}$ in Fig,12). Equipotential lines corresponding to $V_{0} / 2$ are also plotted to represent the fragments immediately after scission. As expected, the part of the wave packet that is emitted has higher average energy and more nodes (a larger quantum number).

In the figures 13 - 14 are shown two wave packets at $\Omega=5 / 2$. The total wave functions are restricted to only one of the fragments. One can see the effect of the centrifugal potential: the wave functions are shifted with respect to the $\rho$ axis. For this reason they cannot be present in the neck region where the potential changes mostly. Their contribution to scission neutron multiplicity is therefore reduced.
[1] N. Carjan, M. Rizea, Phys. Lett. B 747, 178 (2015).

[2] M. Rizea, N. Carjan, Proc. Rom. Acad. A 16, 176 (2015).

[3] R. Capote, N. Carjan, S. Chiba, Phys. Rev. C 93, 024609 (2016).

[4] N. Carjan, M. Rizea, P. Talou, EPJ Web of Conferences 146, 04002 (2017).

[5] S. Lemaire, P. Talou, T. Kawano, M. B. Chadwick, and D. G. Madland, Phys. Rev. C 72, 024601 (2005).

[6] J. Randrup and R. Vogt, Phys. Rev. C 80, 024601 (2009).

[7] O. Litaize and O. Serot, Phys. Rev. C 82, 054616 (2010).

[8] T. Kawano, P. Talou, I. Stetcu, M. B. Chadwick, Nuc. Phys. A 913, 51 (2013).

[9] K.-H. Schmidt, B. Jurado, C. Amouroux, C. Schmitt, Nucl. Data Sheets 131, 107 (2016).

[10] R. Capote, Y.-J. Chen, F.-J. Hambsch, N. V. Kornilov, J. P. Lestone, O. Litaize, B. Morillon, D. Neudecker, S. Oberstedt, T. Ohsawa, N. Otuka, V. G. Pronyaev, A. Saxena, O. Serot, O. A. Shcherbakov, N.-C. Shu, D. L. Smith, P. Talou, A. Trkov, A. C. Tudora et al., Nucl. Data Sheets 131, 1 (2016).

[11] M. Rizea, N. Carjan, Nuc. Phys. A 909, 50 (2013).

[12] F. A. Ivanyuk, K. Pomorski, Phys. Rev. C 79, 054327 (2009).

[13] N. Carjan, P. Talou, O. Serot, Nucl. Phys. A 792, 102
(2007).

[14] A. Bulgac, P. Magiersky, K. J. Roche, I. Stetcu, Phys. Rev. Lett. 116, 122504 (2016).

[15] G.R. Hadley, Opt. Lett. 16, 624 (1991).

[16] M. Rizea, V. Ledoux, M. Van Daele, G. Vanden Berghe, N. Carjan, Comp. Phys. Commun., 179(7), 466 (2008).

[17] M. Rizea, N. Carjan, Commun. Comput. Phys. 9, 917 (2011).

[18] M. Rizea, N. Carjan, Eur. Phys. J. A 52, 368 (2016).

[19] M. Rizea, N. Carjan, Theory-4 Workshop (2017), Varna, Bulgaria, EPJ Web of Conferences 169, 00020 (2018).

[20] A. Göök, F.-J. Hambsch, W. Geertz, M. Vidali, private communication.

[21] A. Göök, F.-J. Hambsch, S. Oberstedt, Theory-4 Workshop (2017), Varna, Bulgaria, EPJ Web of Conferences 169, 00004 (2018).

[22] V.V. Pashkevich, Nucl. Phys. A 169, 275 (1971).

[23] N. Carjan, M. Rizea, Phys. Rev. C 82, 014617 (2010).

[24] N. Kornilov, F.-J. Hambsch at al., Nucl. Sci. Eng. 165, 117 (2010).

[25] J. M. Blatt, V. F. Weisskopf, Theoretical Nuclear Physics (John Wiley, New York, 1958) 368.

[26] N. Kornilov, Fission Neutrons: Experiments, Evaluation, Modeling and Open Problems, Springer, Switzerland (2015) 

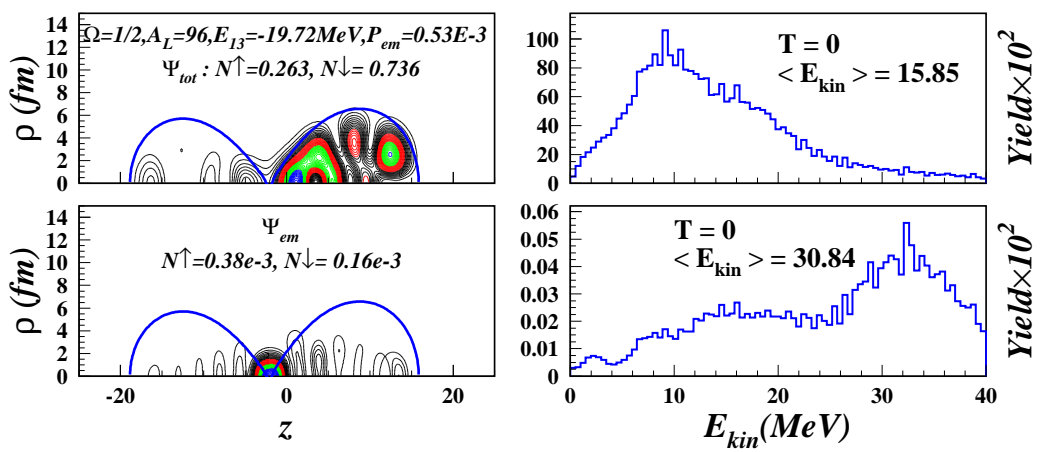

FIG. 10. Square modulus of the total and emitted $\mathrm{WF}_{13}$ at $\Omega=1 / 2$ immediately after scission (left column) and the corresponding energy distributions (right column). $\mathrm{N} \uparrow$ and $\mathrm{N} \downarrow$ are the square moduli of the spin-up and spin-down components.
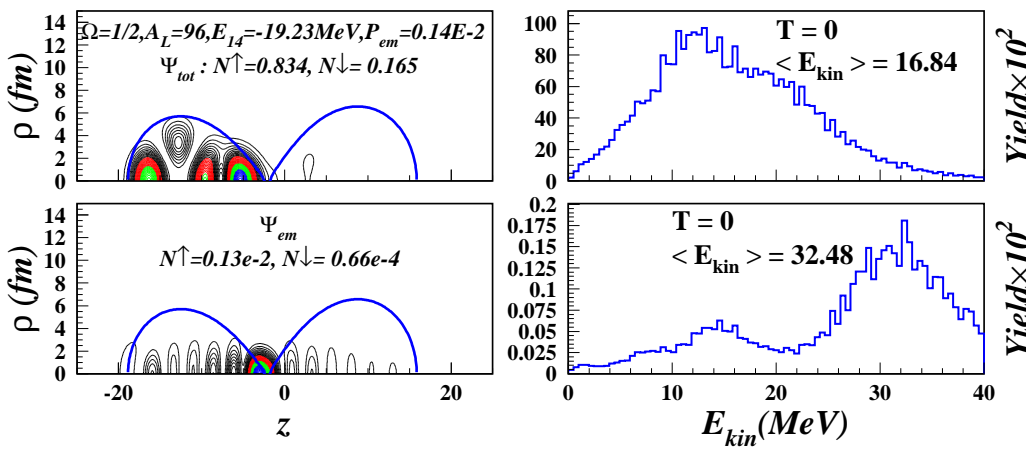

FIG. 11. The same as in Fig.10 but for $\mathrm{WF}_{14}$ at $\Omega=1 / 2$
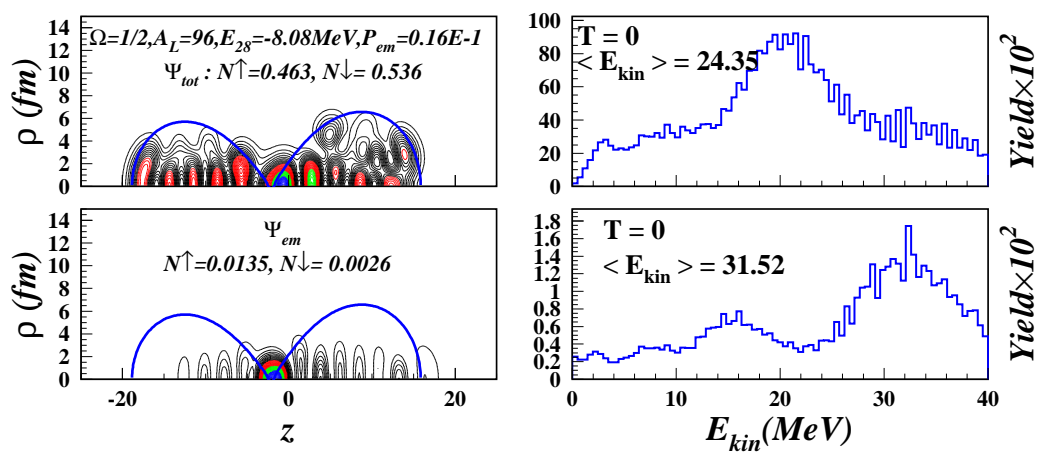

FIG. 12. The same as in Fig 10 but for $\mathrm{WF}_{28}$ at $\Omega=1 / 2$ 

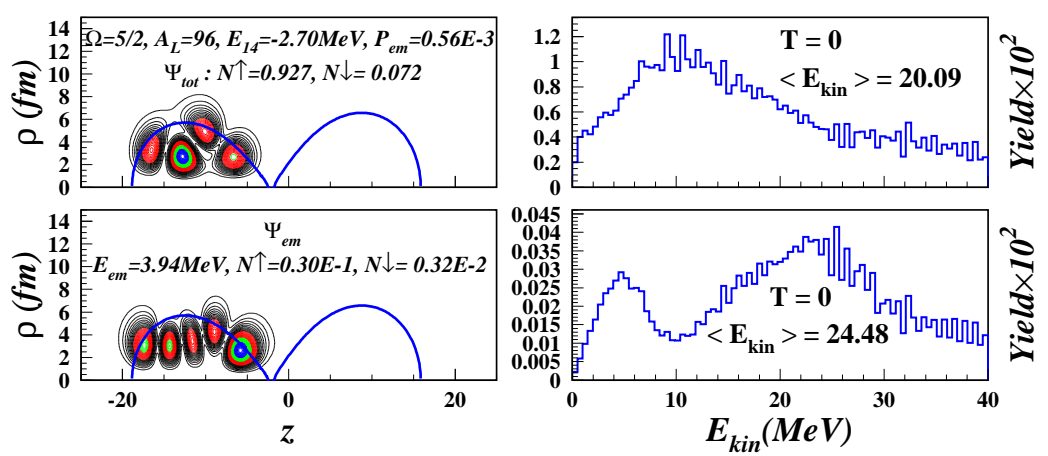

FIG. 13. Square modulus of the total and emitted $\mathrm{WF}_{14}$ at $\Omega=5 / 2$ immediately after scission (left column) and the corresponding energy distributions (right column)
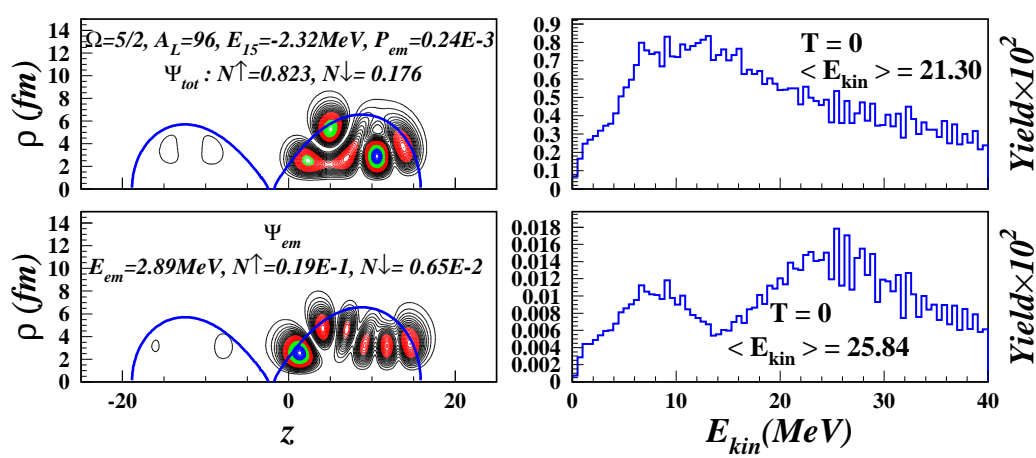

FIG. 14. The same as in Fig. 13 but for $\mathrm{WF}_{15}$ at $\Omega=5 / 2$ 